\title{
Ruídos articulares e sinais de disfunção temporomandibular: um estudo comparativo por meio de palpação manual e vibratografia computadorizada da ATM
}

\section{Joint sounds and signs of temporomandibular disorder: a comparative study by means of manual palpation and computer-based vibrational analysis}

Paulo César Rodrigues CONTI*

João Evandro da Silva MIRANDA**

Flávia ORNELAS***

\footnotetext{
CONTI, P. C. R.; MIRANDA, J. E. da S.; ORNELAS, F. Ruídos articulares e sinais de disfunção temporomandibular: um estudo comparativo por meio de palpação manual e vibratografia computadorizada da ATM. Pesqui Odontol Bras, v. 14 , n. 4 , p. 367-371, out./dez. 2000.
}

\begin{abstract}
Esta pesquisa teve como finalidade estimar a validade interexaminadores, em detectar sons articulares e comparar os resultados com um sistema computadorizado (SONOPAK). Para isto, uma amostra de 45 indivíduos foi selecionada aleatoriamente e dividida em dois grupos. O grupo experimental foi formado por 24 pacientes, que apresentavam problemas articulares, e o grupo controle por 19 pacientes com ausência de qualquer relato ou queixa, compativel com DTM. Sessenta e sete por cento dos pacientes eram mulheres, com médias de idade de 36 anos. Os resultados da eletrovibratografia (EVG) foram comparados com os obtidos pela palpação manual. Todos os examinadores desconheciam o grupo ao qual pertencia o paciente examinado. Para análise dos resultados de concordância, foi utilizado o teste de Cohen's kappa e o percentual de concordância. Os resultados mostraram uma prevalência de 62,5\% e 42,1\% dos sons articulares, apresentados pelo grupo experimental e grupo controle, respectivamente. Pela análise dos resultados, concluiu-se que os sons articulares são comumente apresentados em ambos os grupos, porém sua identificação e classificação são dificeis, mesmo quando obtidos por aparelhos computadorizados. Embora a amostra deste estudo seja pequena, os resultados indicam que a EVG deve ser utilizada com cautela, e a calibração dos examinadores pode melhorar a identificação dos sons articulares.
\end{abstract}

UNITERMO: Articulação temporomandibular.

\section{INTRODUÇÃO}

As disfunções temporomandibulares (DTM) caracterizam-se por uma série de sinais e sintomas, que incluem dores faciais, limitação de movimentação mandibular e ruídos articulares ${ }^{5,10}$.

Considerado por muito tempo como um sinal cardinal e característico das DTM, sabe-se que ruídos articulares da ATM são freqüentes, mesmo numa população assintomática ${ }^{6,20}$.

Dessa forma, análises realizadas por meio de ressonância magnética demonstraram que cerca de $30 \%$ de uma população livre de sintomas apresentam algum tipo de ruído articular ${ }^{12}$.
Entre as causas e alterações estruturais que podem causar algum tipo de som articular, as mais freqüentes são os deslocamentos de disco articular para anterior, alterações estruturais de superficie articular e hipermobilidade do complexo côndilo-disco, além dos processos degenerativos, que provocaram as crepitações ${ }^{7,9,15,16,19,22,24}$.

O relacionamento entre os deslocamentos de disco articular e a sintomatologia dolorosa da ATM ainda não está bem elucidado. Apesar de bastante freqüentes em uma população de pacientes com disfunção, não podem ser considerados como uma condição necessária para iniciar processos inflamatórios intra-articulares e conseqüente dor.

* Professor Doutor do Departamento de Prótese; **Doutorando em Reabilitação Oral; ***Aluna do Curso de Graduação - Faculdade de Odontologia de Bauru da USP. 
CONTI, P. C. R.; MIRANDA, J. E. da S.; ORNELAS, F. Ruídos articulares e sinais de disfunção temporomandibular: um estudo comparativo por meio de palpação manual e vibratografia computadorizada da ATM. Pesqui Odontol Bras, v. 14, n. 4, p. 367-371, out./dez. 2000.

A análise clínica para determinação dos ruídos articulares também é motivo de controvérsia entre os autores. Palpação manual e auscultação através de estetoscópio tem sido os meios mais comumente utilizados, na rotina clínica para análise destes ruídos ${ }^{1,8,11,13,14,17,21}$.

Apesar de consideradas como métodos objetivos, pela maioria dos autores, as técnicas acima descritas apresentam uma série de limitações, quando utilizadas em trabalhos de pesquisa, devido ao caráter subjetivo desta abordagem.

Com objetivo de parametrizar estes achados, aparelhos de sonografia computadorizada têm sido utilizados, para análise de freqüência e amplitude dos sons da ATM, o que possibilita uma diferenciação padronizada entre os diversos tipos de ruídos produzidos, tanto por uma ATM em condições normais como por aquelas articulações acometidas por alterações estruturais.

Tais aparelhos são capazes de captar vibrações estruturais geradas pelo ruído das ATM e reproduzir graficamente esses achados, o que possibilita, além de uma análise mais acurada, um controle da evolução do caso, quando sons articulares fazem parte da queixa principal do paciente.

Portanto, o objetivo deste trabalho foi comparar, através de vibratografia da ATM, a presença e tipo de ruído presente numa população de pacientes de DCM, quando comparada com um grupo controle.

\section{MATERIAL E MÉTODO}

\section{Amostra}

A pesquisa foi realizada utilizando-se uma amostra de 43 indivíduos, divididos em 2 grupos independentemente de sexo e idade. Os grupos (experimental e controle) foram enquadrados nos seguintes critérios:

\section{Grupo I - grupo experimental}

Foi constituído por 24 indivíduos, selecionados aleatoriamente entre os pacientes da Clínica de Dor Orofacial e DCM, do Departamento de Prótese da FOB-USP, que apresentavam como critério de inclusão a presença de sintomas de DCM, de caráter intra- ou extra-articular, ausência prévia de tratamentos irreversiveis, como reabilitação oral ou ajuste oclusal por desgaste seletivo, ausência de patologias sistêmicas, reumáticas ou de procedimentos cirúrgicos da ATM.

\section{Grupo II - grupo controle}

Foi formado por 19 pacientes, escolhidos de forma aleatória nas clínicas de tratamento odontológico da FOB-USP. O critério de inclusão para este grupo foi a ausência de qualquer relato ou queixa compativel com DCM, ausência de tratamentos protéticos extensos e patologias sistêmicas reumáticas.

\section{Exame objetivo}

Toda a amostra foi submetida, além de a exame da musculatura mastigatória e avaliação da ATM por meio de palpação manual seguindo as orientações de PERTES; GROSS ${ }^{18}$, a análise da condição muscular. A ATM foi submetida à palpação dos aspectos lateral e posterior, bem como à análise do seu padrão de movimentação protrusiva e lateral, além de possiveis desvios ou deflexões, durante essa movimentação.

A oclusão dentária foi avaliada com respeito ao número de dentes ausentes e interferências oclusais, nas posições de relação cêntrica e movimentos excursivos da mandibula.

A análise clínica dos ruídos foi realizada por dois examinadores experientes (não calibrados), de maneira "cega", ou seja, sem conhecimento dos resultados do outro examinador, bem como do grupo o qual o indivíduo pertencia. Os resultados obtidos foram assinalados em formulário apropriado.

\section{Análise dos ruídos articulares, por vibração de superfície}

Todos os participantes deste estudo foram submetidos à análise dos ruídos articulares, por meio de vibração de superfície, utilizando-se o sistema de eletrovibratografia (SONOPAK System, Joint Vibration Analysis, BioResearch Inc., Milwalkee, Wiscosin, EUA). Os indivíduos foram orientados a realizar abertura máxima e fechamento da boca, orientados por um sinal sonoro e visual, existente na tela do programa Biopak.

A vibração superficial foi detectada por dois acelerômetros e enviada para um amplificador diferencial. Este sinal, uma vez filtrado, foi transformado em aproximadamente 4.000 pontos, pelo computador acoplado ao sistema.

\section{Análise estatística}

Para análise dos resultados, foram utilizados os testes de Cohen's kappa e percentual de concordância. A prevalência das diversas condições foi expressa em percentuais. 
CONTI, P. C. R.; MIRANDA, J. E. da S.; ORNELAS, F. Ruídos articulares e sinais de disfunção temporomandibular: um estudo comparativo por meio de palpação manual e vibratografia computadorizada da ATM. Pesqui Odontol Bras, v. 14, n. 4, p. 367-371, out./dez. 2000.

\section{RESULTADOS}

A amostra foi formada por 29 pacientes do sexo feminino e 14 do sexo masculino, sendo que no grupo I (com DCM), 79\% (19 pacientes) corresponderam ao sexo feminino e $21 \%$ (5 pacientes), ao masculino. No grupo controle, $52 \%$ (10 pacientes) corresponderam ao sexo feminino e $48 \%$ (9 pacientes), ao masculino.

A média de idade foi de 36 anos, sendo a média dos pacientes com DCM de 33 anos e, do grupo controle, de 31 anos.

No exame de palpação manual, realizado pelo examinador 1 , foi observado estalido simples ou recíproco e/ou crepitação em 53\% (23 pacientes). No grupo de DCM, essa porcentagem foi de $62 \%$ (15 pacientes). No grupo controle, foi de $42 \%$ (8 pacientes). Esses dados podem ser observados na Tabela 1 .

Com os dados obtidos nos exames de palpação e vibratografia computadorizada, foram realizados os testes de Cohen's kappa e teste do percentual de concordância, com os resultados observados na Tabela 2.

\section{DISCUSSÃO}

Os sons articulares são achados comuns mesmo numa população assintomática ${ }^{1,2}$. Assim, a

TABELA 1 - Freqüência de ruídos articulares no grupo 1 (com DCM) e grupo controle.

\begin{tabular}{c|c|c}
\hline Grupo & Suídos & Não \\
\hline DCM & 15 & 9 \\
\hline Controle & 8 & 11 \\
\hline \hline
\end{tabular}

presença de ruídos articulares não necessariamente indica presença de patologias articulares ou disfunção. Também, nesta pesquisa, ao analisar o grupo com DCM, verificou-se que $79 \%$ dos pacientes pertenciam ao sexo feminino, o que concorda com outros estudos epidemiológicos, realizados previamente $^{5,6}$.

Analisando-se os resultados obtidos pela palpação manual, observamos que $53 \%$ do total da amostra se apresentam com ruídos articulares, $62 \%$ pertencentes ao grupo com DCM e $42 \%$ pertencentes ao grupo controle. Esse fato está de acordo com os achados de trabalhos, em que se tem encontrado deslocamentos de disco articular para anterior (associados ou não a ruídos articulares) numa população assintomática ${ }^{19,20,24}$. Tais trabalhos têm utilizado, desde a palpação manual, exames de ressonância magnética e análise computadorizada, sendo que para todos existem dificuldades e limitações, ou seja, ainda não existe uma técnica $100 \%$ precisa, para se diagnosticar algumas condições intra-articulares.

Outro fator a ser questionado é a necessidade de intervenção, quando somente o estalido é detectado. Parece unanimidade que a presença de estalido, na ausência de sintomas não requer tratamento, apesar de esse fator ser mais freqüentemente encontrado numa população com sintomas intra- e extra-articulares, como demonstrado nessa pesquisa e suportado pela literatura ${ }^{20}$. Esse fato é baseado nos achados que afirmam que somente uma pequena parcela da população, que possui deslocamentos de disco, apresentarão algum problema. A recidiva dos ruídos, com o passar do tempo, também contra-indica as terapias voltadas somente para sua eliminação.

TABELA 2 - Distribuição dos achados clinicos dos percentuais de concordância (valores de kappa e percentual de concordância, em \%).

\begin{tabular}{|c|c|c|c|c|c|c|c|c|c|c|c|c|c|c|c|c|}
\hline \multirow{2}{*}{ Exame } & \multicolumn{2}{|c|}{ ES } & \multicolumn{2}{|c|}{ ER } & \multicolumn{2}{|c|}{$\mathrm{C}$} & \multicolumn{2}{|c|}{$\mathrm{H}$} & \multicolumn{2}{|c|}{ DDA R } & \multicolumn{2}{|c|}{ DDA S } & \multicolumn{2}{|c|}{$\mathrm{OA}$} & \multicolumn{2}{|c|}{$\mathrm{HC}$} \\
\hline & Kap & Con & Kap & Con & Kap & Con & Kap & Con & Kap & Con & Kap & Con & Kap & Con & Kap & Con \\
\hline $\begin{array}{l}\text { Ex } 1 \\
\text { Ex } 2\end{array}$ & 0,06 & 69 & 0,31 & 74 & 0,2 & 79 & 0,06 & 60 & 0,25 & 63 & 0 & 88 & 0 & 88 & 0,22 & 65 \\
\hline $\begin{array}{l}\text { Ex } 1 \\
\text { SO }\end{array}$ & 0,14 & 60 & 0,2 & 62 & 0 & 93 & 0,4 & 79 & 0,04 & 48 & 0,1 & 90 & 0 & 90 & 0,13 & 62 \\
\hline $\begin{array}{l}\text { Ex } 2 \\
\text { SO }\end{array}$ & 0,03 & 69 & 0,1 & 58 & 0 & 76 & 0 & 55 & 0 & 32 & 0 & 90 & 0 & 100 & 0,07 & 62 \\
\hline
\end{tabular}

Ex 1 = Examinador $\mathrm{n}^{\circ} 1 ;$ Ex 2 = Examinador $\mathrm{n}^{\circ} 2 ; \mathrm{SO}=$ SONOPAK; ES = Estalido simples; ER = Estalido reciproco; $\mathrm{C}=$ Crepitação; $\mathrm{H}=$ Ruído de hipermobilidade; DDA $\mathrm{R}=$ Deslocamento do disco articular com redução; DDA S = Deslocamento do disco articular sem redução; OA = Osteoartrite; HC = Hipertranslação condilar. 
CONTI, P. C. R.; MIRANDA, J. E. da S.; ORNELAS, F. Ruídos articulares e sinais de disfunção temporomandibular: um estudo comparativo por meio de palpação manual e vibratografia computadorizada da ATM. Pesqui Odontol Bras, v. 14, n. 4, p. 367-371, out./dez. 2000.

Em relação à técnica de detecção, parece-nos que a implementação de um sistema computadorizado não otimizou os resultados. Os valores do teste de kappa foram abaixo da média aceitável de 0,4 , apesar do percentual de concordância ter sido razoável, para a maioria dos tipos de ruidos.

Este fato deve-se basicamente a dois fatores: o primeiro seria a presença de algumas condições, como osteoartrite da ATM e deslocamentos de disco, mais raras na população de DCM, o que aumentaria a chance de concordância por acaso, e diminuiria o valor do teste de kappa. O segundo seria a dificuldade, natural das ciências biológicas, em se padronizar e calibrar os examinadores. Aceita-se, em Medicina, que dificilmente se consegue valores de kappa maiores que 0,35, independentemente da condição que está sendo avaliada.

Mesmo com calibração, a concordância interexaminadores para detecção de ruídos articulares tem sido bastante baixa.

No presente trabalho, os melhores valores foram obtidos para análise de estalido recíproco (entre examinadores 1 e 2) e para ruídos de hipermobilidade (entre examinador 1 e SONOPAK) com valores próximos do aceitável: 0,31 e 0,40, respectivamente.

O diagnóstico da hipermobilidade foi em muito facilitado pelo fato de a mesma sempre ocorrer em estágios finais de abertura máxima da boca, aumentando os indices de concordância.

Pode-se concluir, portanto, que existe uma necessidade de calibração rigorosa intra- e interexaminadores, para que se possam padronizar resultados de estudos clínicos e epidemiológicos.

Em relação à utilização do SONOPAK, parece-nos que não existe uma indicação de sua utilização, como rotina, na prática diária. Eventualmente, alguns dados, como freqüência e duração dos ruídos possam ser úteis em algumas pesquisas. Porém, deve ficar claro que tais aparelhos podem causar muitos "falso-positivos" e gerar um sobretratamento, por parte do profissional, em condições autolimitantes e não progressivas, o que seria catastrófico, do ponto de vista econômico, biológico e até mesmo, ético. Existe também uma série de dificuldades técnicas na utilização desses aparelhos, que limitam sua utilização. Assim, cautela é necessária, em relação ao marketing que se faz, em relação ao eletrodiagnóstico, nas DCM.

\section{CONCLUSÕES}

1. Os ruídos articulares são mais freqüentemente encontrados numa população com sintomas de DCM.

2. O sistema SONOPAK não contribuiu para o diagnóstico, quando comparado ao exame clínico.

3. Foi baixa a concordância interexaminadores, sem prévia calibração entre os mesmos.

CONTI, P. C. R.; MIRANDA, J. E. da S.; ORNELAS, F. Joint sounds and signs of temporomandibular disorder: a comparative study by means of manual palpation and computer-based vibrational analysis. Pesqui Odontol Bras, v. 14, n. 4, p. 367-371, out./dez. 2000.

Both vibrational analysis and clinical examination have been claimed to identify intracapsular temporomandibular joint (TMJ) disorders, particularly disc displacements. The purpose of this study was to evaluate the interexaminer consensus on detecting TMJ sounds and to compare this with the results obtained through a computerized system (SONOPAK). The sample was composed of 45 people and it was divided in 2 groups: an experimental group of 24 patients presenting with TMJ complaints (sounds and/or pain), and a control group of 19 individuals with no obvious signs or symptoms of temporomandibular disorders. Sixty-seven percent of the patients were females with a mean age of 36 years. The control group was gender- and age-matched with the test group. Temporomandibular joint electrovibratography (JVA) was performed on the whole sample by one examiner. This was followed by light manual palpation of the TMJs by 2 examiners, who were not calibrated and were not aware of the results of the previous vibratographic (EAV) examination. Both examiners were unaware of which group was being examined. Indices of agreement and the Cohen's kappa test were used to analyze the data. A prevalence of $62.5 \%$ and $42.1 \%$ of TMJ sounds was found for the experimental and control groups, respectively. Indices of agreement ranged from $32 \%$ to $100 \%$ and kappa index ranged from 0 to 0.4 for all conditions investigated (opening and reciprocal clicking, crepitation, and terminal thud). Although acceptable agreement was achieved, the values for kappa index were considered poor for all variables (fair agreement is considered between 0.4 and 0.75). Temporomandibular joint sounds are commonly found in both patients and nonpatients, but their detection and classification are difficult, even when an electronic device is employed. Although the sample in this study was small, the results indicate that electrovibratography (JVA) should be used with caution, and that interexaminer calibration may improve the identification of joint sounds.

UNITERM: Temporomandibular joint. 
CONTI, P. C. R.; MIRANDA, J. E. da S.; ORNELAS, F. Ruídos articulares e sinais de disfunção temporomandibular: um estudo comparativo por meio de palpação manual e vibratografia computadorizada da ATM. Pesqui Odontol Bras, v. 14, n. 4, p. 367-371, out./dez. 2000.

\section{REFERÊNCIAS BIBLIOGRÁFICAS}

1. BRACCO, P.; DEREGIBUS, A.; PISCETTA, R. et al. Clicking: a comparison of clinical examination, sonography and paxiography. J Craniomandib Pract, v. 15, n. 2, Apr. 1997.

2. CHRISTENSEN, L. V.; DONEGAN, S. J.; MCKAY, D. C. Temporomandibular joint vibration analysis in a sample of nonpatients. J Craniomandib Pract, v. 10, n. 1, p. 35-42, 1992.

3. CHRISTENSEN, L. V.; ORLOFF, J. Reproducibility of temporomandibular joint vibrations (eletrovibratography). J Oral Rehabil, v. 19, p. 253-263, 1992.

4. CHRISTENSEN, L. V. Physics and the sounds produced by the temporomandibular joints. Part II. J Oral Rehabil, v. 19 , p. 615-627, 1992.

5. CONTI, P. C. R.; BONACHELA, W. C.; ARAUJO, C. R. P. TMD symptoms in short dental arch patients. Anais da "22nd Meeting on Temporomandibular Disorders and Orofacial Pain", San Diego, CA, 1997.

6. CONTI, P. C. R.; FERREIRA, P. M.; PEGORARO, L. F. et al. A cross-sectional study of prevalence and etiology of signs and symptoms of temporomandibular disorders in high school and university students. J Orofac Pain, v. 10, p. 254-262, 1996.

7. GAY, T.; BERTOLAMI, C. N.; DONOFF, B. et al. The acoustical characteristics of the normal and abnormal temporomandibular joint. J Oral Maxilofac Surg, v. 45, p. 397-407, 1987.

8. GAY, T.; BERTOLAMI, C. N. The spectral properties of temporomandibular joint sounds. J Dent Res, v. 66, n. 6, p. 1189-1194, 1987.

9. HEFFEZ, L.; BLAUSTEIN, D. Advances in sonography of the temporomandibular joint. Oral Surg Oral Med Oral Pathol, v. 62, p. 486-495, 1986.

10. ISHIGAKI, S.; BESSETTE, R. W.; MARUYAMA, T. A clinical study of temporomadibular joint (TMJ) vibration analysis for internal derangement and/or degenerative joint dislease. J Craniomandibular Pract, v. 11, p. 7-13, 1992.

11. Diagnostic accuracy of TMD vibration analysis for internal derangement and/or degenerative joint dislease. J Craniomandib Pract, v. 12, n. 4, p. 241-246, Oct. 1994

12. Vibration analysis of the temporomandibular joints with meniscal displacement with and without reduction. J Craniomandib Pract, v. 11, n. 3, p. 192-201, July 1993.

13. Vibration analysis of the temporomandibular joints with degenerative joint disease. J Craniomandib Pract, v. 11, n. 4 , p. 276-283, 1993.

14. Vibration of the temporomandibular joints with normal radiographic images: comparison between asymptomatic patients. J Craniomandib Pract, v. 11, n. 2, p. 88-94, Apr. 1993.

15. MOTOYOSHI, M.; OHYA, M.; HASEGAMA, M. et al. A study of temporomandibular joint sounds. Part. 1. Relationship with articular disc displacements. J Nihon Univ Sch Dent, v. 36, n. 1, p. 48-51, 1994.

16. OKESON, J. P. Orofacial Pain: Guidelines for assessment, diagnosis, and management. Quintessence Books, 1995.

17. PENTTI, J. A.; PENTTI, K. K. Disorders in TMJ research. J Craniomandib Disord Facial Oral Pain, v. 4, p. 223-227, 1992.

18. PERTES, R. A.; GROSS, S. G. Clinical management of temporomandibular disorders and orofacial pain. Quintessence Books, 1995.

19. POLLMANN, L.; DOZ, P. Sounds produced by the mandibular joint in a sample of healthy workers. J Orofac Pain, v. 7, p. 359-361, 1993.

20. RIBEIRO, R. F.; TALLENTS, R. S.; KATZBERG, R. W. et al. The prevalence of disc displacement in symptomatic and asymptomatic volunteers aged 6 to 25 years. J Orofac Pain, v. 11, p. 37-47, 1997.

21. YOSHIDA, H.; SANO, T.; KATAOKA, R. et al. A preliminary investigation of a method of detecting temporomandibular joint sounds. J Orofac Pain, v. 8, p. 73-79, 1994.

22. WABEKE, K. B.; SPRIRYT, R. J.; ZAAG, V. D. The reliability of clinical methods for recording temporomandibular joint sounds. J Dent Res, v. 73, n. 6, p. 1157-1162, 1994.

23. WESTHING, L. Occlusal interferences in retruded contact position in a sample of healthy workers. J Orofac Pain, v. 7, n. 4, p. 359-361, 1993.

24. WIDMALM, S.; WESTNSSON, P. L.; BROOKS, S. L. et al. Temporomandibular joints sounds: correlation to joint structure in fresh autopsy specimens. Am J Orthod Dentofac Orthop, v. 101, p. 60-69, 1992.

Recebido para publicação em 28/09/99 Enviado para reformulação em 06/04/00 Aceito para publicação em 21/07/00 